\title{
Quaternary Diffusion Coefficients in a Protein-Polymer-Salt-Water System Determined by Rayleigh Interferometry
}

\author{
Onofrio Annunziata, ${ }^{*, \dagger}$ Alessandro Vergara,${ }^{\ddagger}$ Luigi Paduano, ${ }^{\ddagger}$ Roberto Sartorio,, \\ Donald G. Miller, ${ }^{\S}$ and John G. Albright ${ }^{\dagger}$ \\ Department of Chemistry, Texas Christian University, Fort Worth, Texas 76129, Department of Chemistry, \\ "Federico II" University of Naples, Naples, 80126, Italy, and Geosciences and Environmental Technologies, \\ Lawrence Livermore National Laboratory, Livermore, California 94551
}

Received: July 22, 2009; Revised Manuscript Received: August 11, 2009

\begin{abstract}
We have experimentally investigated multicomponent diffusion in a protein-polymer-salt-water quaternary system. Specifically, we have measured the nine multicomponent diffusion coefficients, $D_{i j}$, for the lysozyme-poly(ethylene glycol)- $\mathrm{NaCl}$-water system at $\mathrm{pH} 4.5$ and $25{ }^{\circ} \mathrm{C}$ using precision Rayleigh interferometry. Lysozyme is a model protein for protein-crystallization and enzymology studies. We find that the protein diffusion coefficient, $D_{11}$, decreases as polymer concentration increases at a given salt concentration. This behavior can be quantitatively related to the corresponding increase in fluid viscosity only at low polymer concentration. However, at high polymer concentration $(250 \mathrm{~g} / \mathrm{L})$, protein diffusion is enhanced compared to the corresponding viscosity prediction. We also find that a protein concentration gradient induces salt diffusion from high to low protein concentration. This effect increases in the presence of poly(ethylene glycol). Finally, we have evaluated systematic errors associated with measurements of protein diffusion coefficients by dynamic light scattering. This work overall helps characterize protein diffusion in crowded environments and may provide guidance for further theoretical developments in the field of protein crystallization and protein diffusion in such crowded systems, such as the cytoplasm of living cells.
\end{abstract}

\section{Introduction}

Diffusion in liquid mixtures containing proteins has been investigated for its importance in a wide range of disciplines. For instance, this transport property is studied for understanding and optimizing the production of protein crystals. ${ }^{1,2}$ Such crystals are critical for the determination of protein three-dimensional structures, ${ }^{3}$ protein purification, ${ }^{4}$ and the preparation of crosslinked enzyme crystals relevant to the catalysis of petroleum derivatives in nonaqueous media., ${ }^{5,6}$ Diffusion is also an important aspect of all biochemical, medical, and manufacturing processes in which concentration gradients occur. ${ }^{7,8}$ Furthermore, applications in which convection is minimized with respect to diffusion by employing microgravity, gels, capillaries, and microfluidic devices, are of increasing importance. ${ }^{2,9}$ This is because diffusion, compared to convection, is a more controllable and reproducible process. Effective modeling, prediction, and design of these processes require accurate description of protein transport.

One source of complexity in protein aqueous solutions is the presence of other solutes such as salts, polymers, and osmolytes. ${ }^{10-12}$ These additives are usually used to induce phase transformations such as protein crystallization, ${ }^{13-16}$ liquid-liquid phase separation, aggregation, and gelation. ${ }^{17-20}$ They are also used to stabilize protein native structure ${ }^{12}$ and modulate enzymatic activity in aqueous and nonaqueous media. ${ }^{21}$

For a system with $N+1$ components, isothermal diffusion can be described using the generalized Fick's first law: $:^{22}$

* To whom correspondence should be addressed. Phone: (817) 257-6215. Fax: (817) 257-5851. E-mail: o.annunziata@tcu.edu.

$\uparrow$ Texas Christian University.

* "Federico II" University of Naples.

$\S$ Lawrence Livermore National Laboratory (retired).

$$
J_{i}=-\sum_{j=1}^{N} D_{i j} \nabla C_{j} \quad(\text { with } i=1,2, \ldots, N)
$$

Here, $C_{i}$ is the molar concentration of solute $i$, and $J_{i}$ is its corresponding flux. In eq 1 , the $D_{i j}$ are multicomponent diffusion coefficients. Each main-term diffusion coefficient, $D_{i i}$, links the flux of solute $i$ to its own concentration gradient, while each cross-term diffusion coefficient, $D_{i j}(i \neq j)$, links the flux of solute $i$ to the gradient of solute $j$. According to eq 1 , four diffusion coefficients are required for a ternary system ${ }^{23}$ and nine for a quaternary system. ${ }^{24}$ Both $J_{i}$ and $D_{i j}$ depend on the reference frame (see below)..$^{25,26}$

Due to the lack of data, diffusion in multicomponent systems has usually been approximated by neglecting cross-diffusion terms. However, this approximation can lead to inaccurate descriptions of transport processes. ${ }^{27}$ For protein crystallization in the presence of precipitants, neglecting cross terms can lead to the erroneous conclusion that the concentrations of precipitants are almost the same throughout the solution, including up to the crystal interface. However, when the cross-term diffusion coefficients are included, model calculations show that there can be a variation of several percent in the precipitant solute concentration at the crystal surface, which can change as a function of time. ${ }^{28}$ Such variation with time can increase precipitant concentration at the crystal interface. This increases local supersaturation and promotes precipitant inclusion inside the crystal, thereby potentially compromising crystal quality.

Several experimental investigations on multicomponent diffusion in ternary aqueous protein systems have been reported..$^{23,29-34}$ Diffusion measurements at $25^{\circ} \mathrm{C}$ on lysozymesalt-water ${ }^{31,32}$ and lysozyme-poly(ethylene glycol) - water $^{33,34}$ by optical interferometry have shown that cross-diffusion terms 
are significant. Furthermore, knowledge of these cross terms has enabled the extraction of thermodynamic parameters describing the net interaction between protein and precipitant. ${ }^{29}$

However, many protein-precipitation protocols involve both the use of salts and polymer additives. ${ }^{13}$ Thus, multicomponent diffusion coefficients for four-component protein systems are important. As noted above, this requires the determination of nine diffusion coefficients. We note that only a few experimental investigations have been previously reported on quaternary systems $^{24,35,36}$ due to the demanding experimental effort and the high-precision instrumentation needed for the determination of the nine diffusion coefficients.

We used the precision Gosting diffusiometer operating in the Rayleigh interfometric mode to measure the nine diffusion coefficients for the lysozyme-tetra(ethylene glycol)- $\mathrm{NaCl}$-water system at $\mathrm{pH} 4.5$ and $25{ }^{\circ} \mathrm{C} .{ }^{24}$ This investigation, reported in ref 24 , represented until now the most successful experimental determination of diffusion coefficients in a quaternary system, and it demonstrates the feasibility of diffusion studies on quaternary systems relevant to macromolecules.

Within the context of protein crystallization, poly(ethylene glycol) (PEG) additives with a significantly higher molecular weight than tetra(ethylene glycol) (TEG) are typically employed in precipitation protocols. Importantly, polymer molecular weight plays a critical role on the effectiveness of PEG as precipitating agent, since its main action on protein solutions can be attributed to an increase in protein-protein net attraction due to depletion interactions or macromolecular crowding. ${ }^{15,34,37-40}$ Thus, further precision studies with PEG of higher molecular weight are needed.

In this paper, we report multicomponent diffusion coefficients for the lysozyme-PEG- $\mathrm{NaCl}$-water quaternary system at $\mathrm{pH}$ 4.5 and $25^{\circ} \mathrm{C}$ at the same low protein concentration of $0.6 \mathrm{mM}$ and for three different precipitant compositions. In the present work, PEG has an average molecular weight of $2000 \mathrm{~g} \mathrm{~mol}^{-1}$ (PEG2000). This molecular-weight PEG has been extensively used in precipitation protocols at both low and high polymer concentrations. ${ }^{13}$ Therefore, our work represents a significant contribution to the understanding of protein diffusion in such crowded environments. Since proteins are typically charged in solution, salts (including buffer components) must be added to screen long-range repulsive electrostatic interactions. ${ }^{11}$ In this way, PEG can effectively induce protein-protein attractive interactions. Thus, these experimental studies must necessarily be performed on quaternary systems containing protein, salt, polymer, and solvent components.

Our main objective is to compare the multicomponent diffusion coefficients obtained for the quaternary system with those obtained for the lysozyme- salt, ${ }^{23}$ lysozyme-PEG, ${ }^{34}$ and PEG-salt aqueous ternary systems in the same experimental conditions. This experimental comparison is critical for designing models to predict diffusion coefficients in complex multicomponent mixtures based on ternary diffusion coefficients, which are experimentally more accessible. Although there are several ternary-diffusion studies on PEG-salt-water systems, ${ }^{41-43}$ ternary diffusion coefficients matching the experimental conditions of our quaternary system were not available. Therefore, we also report here the corresponding ternary diffusioncoefficient measurements for the PEG2000- NaCl-water system.

\section{Experimental Section}

Materials. Hen egg-white lysozyme, recrystallized six times and lyophilized, was purchased from Seikagaku America and used without further purification. The molecular mass of lysozyme solute was taken to be $14307 \mathrm{~g} \mathrm{~mol}^{-1}$, and this value was used to calculate all molar concentrations, after correction for the moisture and chloride content. Poly(ethylene glycol) with average molecular weight of about $2000 \mathrm{~g} \mathrm{~mol}^{-1}$ (PEG2000) was purchased from Aldrich (lot $06423 \mathrm{HZ}$; polydispersity = 1.06) and used without further purification. The molecular mass of PEG2000 solute was taken to be $2000 \mathrm{~g} \mathrm{~mol}^{-1}$, and this value was used to calculate all molar concentrations. $\mathrm{NaCl}$ was purchased from Aldrich (purity listed as 99.9\%), dried by heating at $450{ }^{\circ} \mathrm{C}$ as recommended by $\operatorname{Rard}^{44}$ and used without further purification. Its crystal density was taken to be $2.165 \mathrm{~g} \mathrm{~cm}^{-3}$ for buoyancy corrections. ${ }^{23}$ Deionized water was distilled and then passed through a four-stage Millipore filter system to provide high-purity water for all the experiments. The molar masses of $\mathrm{NaCl}$ and water were taken to be 58.443 and 18.015 $\mathrm{g} \mathrm{mol}^{-1}$, respectively.

Preparation of Solutions. All solutions were prepared by weight, using a Mettler Toledo model AT400 electro balance. The quaternary solutions used for diffusion and viscosity experiments were prepared using dry $\mathrm{NaCl}$ and stock solutions for both lysozyme-water and PEG2000-water. A detailed description of the preparation of solutions has been given elsewhere. ${ }^{23}$

Density Measurements. All density measurements were performed with a Mettler-Paar model DMA40 density meter that was thermostatted with water from a large, well-regulated $\left( \pm 0.01{ }^{\circ} \mathrm{C}\right)$ water bath. This instrument is interfaced to a computer for time averaging and gives a precision of $\pm 2 \times$ $10^{-5} \mathrm{~g} \mathrm{~cm}^{-3}$ or better. ${ }^{23}$

Viscosity Measurements. Viscosity measurements were performed on both the binary system PEG2000- $\mathrm{H}_{2} \mathrm{O}$ and the ternary system PEG2000- $\mathrm{NaCl}-\mathrm{H}_{2} \mathrm{O}$, using an Ubbelhode viscometer in a bath at $25.00 \pm 0.01{ }^{\circ} \mathrm{C}$, with the viscosity of water as a reference.

Free-Diffusion Measurements. Multicomponent diffusion measurements were made with the high-precision Gosting diffusiometer ${ }^{45}$ operated in its Rayleigh interferometric mode. ${ }^{46}$ This interferometer consists of a $\sim 9$-m optical bench $(5000 \mathrm{~kg}$ ) with vibration isolation and several optical and mechanical components. The light source for generating the Rayleigh interference pattern is a $\mathrm{He}-\mathrm{Ne}$ Uniphase laser (543.5 nm, 5 $\mathrm{mW}$ ). The lens components are the main lens (two-element airspaced achromat, focal length: $145 \mathrm{~cm}$ ) and the cylinder lens (two plano-convex lenses, focal length: $68 \mathrm{~cm}$ ). A cell holder is located between these two lens components inside a water bath. The temperature of the bath was regulated at $25.00{ }^{\circ} \mathrm{C}$ with a model PTC-41 Tronac temperature controller to a precision of $\pm 0.001{ }^{\circ} \mathrm{C}$. The cell holder supports a Tiselius cell (where macroscopic-gradient diffusion occurs) and a mask. This mask splits the laser beam into two parts: one going through the diffusion channel of the Tiselius cell and one passing through the water bath (reference channel). The main lens focuses the light source and the cylinder lens focuses the center of the diffusion channel at the detector, where the Rayleigh interference pattern is observed. Data from the Rayleigh interference patterns were collected with a linear charge-coupled device (CCD) array (6000 pixel, $10 \mu \mathrm{m} \times 10 \mu \mathrm{m}$ pixels), mounted vertically on a precision stage. This stage with its vertical array was stepped horizontally through the two-dimensional interference pattern to collect the fringe-position data necessary to calculate the diffusion coefficients. Horizontal positions were obtained with an optical encoder of $\pm 0.5 \mu \mathrm{m}$ accuracy. Data acquisition was controlled via computer, which also performed the subsequent data reduction. ${ }^{47}$ In brief, a typical diffusion experiment using the Gosting diffusiometer starts from preparing a sharp boundary 
TABLE 1: Quaternary Diffusion Data for Lysozyme (1) + PEG2000 (2) $+\mathrm{NaCl}(3)+\mathrm{H}_{2} \mathrm{O}$

\begin{tabular}{|c|c|c|c|}
\hline $\bar{C}_{1}(\mathrm{mM})$ & 0.6000 & 0.5999 & 0.5997 \\
\hline $\bar{C}_{2}(\mathrm{M})$ & 0.05000 & 0.04999 & 0.1249 \\
\hline $\bar{C}_{3}(\mathrm{M})$ & 0.5000 & 0.8998 & 0.4997 \\
\hline $\bar{d}\left(\mathrm{~g} \mathrm{~cm}^{-3}\right)$ & $1.03596_{3}$ & $1.05153_{2}$ & $1.06039_{8}$ \\
\hline$H_{1}\left(\mathrm{~kg} \mathrm{~mol}^{-1}\right)$ & $4.03_{9}$ & $4.15_{0}$ & $4.11_{2}$ \\
\hline$H_{2}\left(\mathrm{~kg} \mathrm{~mol}^{-1}\right)$ & $0.331_{5}$ & $0.313_{2}$ & $0.325_{8}$ \\
\hline$H_{3}\left(\mathrm{~kg} \mathrm{~mol}^{-1}\right)$ & $0.0393_{0}$ & $0.0387_{2}$ & $0.0384_{9}$ \\
\hline $\bar{V}_{0}\left(\mathrm{dm}^{3} \mathrm{~mol}^{-1}\right)$ & 0.01806 & 0.01804 & $0.0180_{5}$ \\
\hline $\bar{V}_{1}\left(\mathrm{dm}^{3} \mathrm{~mol}^{-1}\right)$ & $10.3_{0}$ & $10.1_{7}$ & $10.2_{2}$ \\
\hline $\bar{V}_{2}\left(\mathrm{dm}^{3} \mathrm{~mol}^{-1}\right)$ & $1.67_{3}$ & $1.68_{9}$ & $1.67_{8}$ \\
\hline $\bar{V}_{3}\left(\mathrm{dm}^{3} \mathrm{~mol}^{-1}\right)$ & $0.0191_{9}$ & $0.0197_{5}$ & $0.0199_{9}$ \\
\hline$R_{1}\left(\mathrm{mM}^{-1}\right)$ & 129.0 & 128.8 & 128.9 \\
\hline$R_{2}\left(\mathrm{M}^{-1}\right)$ & 12280 & 12260 & 12380 \\
\hline$R_{3}\left(\mathrm{M}^{-1}\right)$ & 442.5 & 429.8 & 431.3 \\
\hline$\lambda_{1}\left(10^{-9} \mathrm{~m}^{2} \mathrm{~s}^{-1}\right)$ & 0.0498 & 0.0462 & 0.0177 \\
\hline$\lambda_{2}\left(10^{-9} \mathrm{~m}^{2} \mathrm{~s}^{-1}\right)$ & 0.180 & 0.166 & 0.185 \\
\hline$\lambda_{3}\left(10^{-9} \mathrm{~m}^{2} \mathrm{~s}^{-1}\right)$ & 1.096 & 1.115 & 0.697 \\
\hline$\left(D_{11}\right)_{V}\left(10^{-9} \mathrm{~m}^{2} \mathrm{~s}^{-1}\right)$ & $0.0515 \pm 0.0004$ & $0.0481 \pm 0.0005$ & $0.0200 \pm 0.0005$ \\
\hline$\left(D_{12}\right)_{V}\left(10^{-9} \mathrm{~m}^{2} \mathrm{~s}^{-1}\right)$ & $0.00097 \pm 0.00008$ & $0.00096 \pm 0.00008$ & $0.0008 \pm 0.0001$ \\
\hline$\left(D_{13}\right)_{V}\left(10^{-9} \mathrm{~m}^{2} \mathrm{~s}^{-1}\right)$ & $0.00009 \pm 0.00001$ & $0.00009 \pm 0.00001$ & $0.00008 \pm 0.00002$ \\
\hline$\left(D_{21}\right)_{V}\left(10^{-9} \mathrm{~m}^{2} \mathrm{~s}^{-1}\right)$ & $0.20 \pm 0.01$ & $0.21 \pm 0.01$ & $0.54 \pm 0.01$ \\
\hline$\left(D_{22}\right)_{V}\left(10^{-9} \mathrm{~m}^{2} \mathrm{~s}^{-1}\right)$ & $0.199 \pm 0.002$ & $0.195 \pm 0.002$ & $0.231 \pm 0.003$ \\
\hline$\left(D_{23}\right)_{V}\left(10^{-9} \mathrm{~m}^{2} \mathrm{~s}^{-1}\right)$ & $0.0083 \pm 0.0002$ & $0.0078 \pm 0.0002$ & $0.0147 \pm 0.0003$ \\
\hline$\left(D_{31}\right)_{V}\left(10^{-9} \mathrm{~m}^{2} \mathrm{~s}^{-1}\right)$ & $12.1 \pm 0.8$ & $17.1 \pm 0.9$ & $9 \pm 1$ \\
\hline$\left(D_{32}\right)_{V}\left(10^{-9} \mathrm{~m}^{2} \mathrm{~s}^{-1}\right)$ & $2.10 \pm 0.08$ & $3.48 \pm 0.09$ & $1.5 \pm 0.2$ \\
\hline$\left(D_{33}\right)_{V}\left(10^{-9} \mathrm{~m}^{2} \mathrm{~s}^{-1}\right)$ & $1.075 \pm 0.004$ & $1.084 \pm 0.005$ & $0.648 \pm 0.008$ \\
\hline$\left(D_{11}\right)_{0}\left(10^{-9} \mathrm{~m}^{2} \mathrm{~s}^{-1}\right)$ & 0.0522 & 0.0489 & 0.0210 \\
\hline$\left(D_{12}\right)_{0}\left(10^{-9} \mathrm{~m}^{2} \mathrm{~s}^{-1}\right)$ & 0.00123 & 0.0012 & 0.0011 \\
\hline$\left(D_{13}\right)_{0}\left(10^{-9} \mathrm{~m}^{2} \mathrm{~s}^{-1}\right)$ & 0.00011 & 0.00011 & 0.00011 \\
\hline$\left(D_{21}\right)_{0}\left(10^{-9} \mathrm{~m}^{2} \mathrm{~s}^{-1}\right)$ & 0.26 & 0.28 & 0.75 \\
\hline$\left(D_{22}\right)_{0}\left(10^{-9} \mathrm{~m}^{2} \mathrm{~s}^{-1}\right)$ & 0.220 & 0.218 & 0.300 \\
\hline$\left(D_{23}\right)_{0}\left(10^{-9} \mathrm{~m}^{2} \mathrm{~s}^{-1}\right)$ & 0.0103 & 0.0098 & 0.0209 \\
\hline$\left(D_{31}\right)_{0}\left(10^{-9} \mathrm{~m}^{2} \mathrm{~s}^{-1}\right)$ & 12.7 & 18.3 & 10 \\
\hline$\left(D_{32}\right)_{0}\left(10^{-9} \mathrm{~m}^{2} \mathrm{~s}^{-1}\right)$ & 2.31 & 3.89 & 1.8 \\
\hline$\left(D_{33}\right)\left(10^{-9} \mathrm{~m}^{2} \mathrm{~s}^{-1}\right)$ & 1.094 & 1.120 & 0.673 \\
\hline
\end{tabular}

between two uniform solutions of slightly different solute concentrations located inside a vertical channel, then following the increasing separation of the fringes with time. The diffusion coefficients are reported at the average composition. Experimental details on data analysis can be found in ref 24 and references therein.

\section{Results and Discussion}

Diffusion can be described relative to different reference frames. ${ }^{25,26} \mathrm{We}$ report isothermal diffusion coefficients for the volume- and solvent-fixed frames. In the volume-fixed frame, the fluxes of the components of a quaternary system satisfy $\sum_{i=0}^{3}$ $\left(J_{i}\right)_{V} \bar{V}_{i}=0$. Here, $J_{i}$ and $\bar{V}_{i}$ are the molar flux and partial molar volume of component $i$, respectively. The subscript $V$ denotes the volume-fixed frame. The subscript 0 denotes the solvent component when appended directly to a flux and denotes the solvent-fixed frame when appended outside the parentheses to an already-subscripted flux or diffusion coefficient. Subscripts 1,2 , and 3 refer to the lysozyme, PEG2000, and $\mathrm{NaCl}$ components, respectively. Since concentration differences are small and volume changes on mixing are negligible, our interferometric measurements correspond, to an excellent approximation, to the volume-fixed frame. ${ }^{25}$ Volume-fixed quaternary diffusion coefficients will be denoted by $\left(D_{i j}\right)_{V}$ with $i, j$ $=1,2,3$. The corresponding ternary diffusion coefficients will be denoted by $\left(D_{i j}\right)_{V}^{(i j)}$, where the superscript $(i j)$ is introduced outside the parentheses in order to identify the pair of solutes of the ternary system. We note that $\left(D_{i j}\right)_{V}$ at $C_{k}=0$ with $k \neq$ $i, j$ coincides with $\left(D_{i j}\right)_{V}^{(i j)}$.

The $\left(D_{i j}\right)_{V}$ values for the lysozyme + PEG2000 $+\mathrm{NaCl}+$ water system and the eigenvalues, $\lambda_{i}$, of the diffusion-coefficient matrix are reported in Table 1 at three compositions, specified by the overall average concentrations, $\bar{C}_{i}$. Corresponding solventframe coefficients, $\left(D_{i j}\right)_{0}$, are also included (equations for calculation of the $\left(D_{i j}\right)_{0}$ are reported in ref 26). The errors shown for $\left(D_{i j}\right)_{V}$ are approximately 4 times the standard error of the coefficients as determined from the propagation-of-error equations using the full covariance matrix of the least-squares parameters described in ref 24 and references therein. The errors for $\left(D_{i j}\right)_{0}$ (not shown) should be taken to be the same as those for $\left(D_{i j}\right)_{V}$. In all cases, the lysozyme molar concentration is 0.6 $\mathrm{mM}$, which is significantly lower than PEG2000 and $\mathrm{NaCl}$ molar concentrations. Table 1 also contains density data represented by the density parameter, $\bar{d}$, at the average composition and the parameters, $H_{i} \equiv\left(\partial d / \partial C_{i}\right)_{T, p, C_{i}, j \neq i}$, obtained by least-squares calculations from the experimental densities using the linear Taylor series: $d=\bar{d}+\sum_{i=1}^{3} H_{i}\left(C_{i}-\bar{C}_{i}\right)$. The partial molar volumes, $\bar{V}_{i}$, were calculated from these density data as described in ref 46. Finally, the $R_{i}$ values in Table 1 (the refractive index increments) are instrumental constants directly proportional to the derivatives of refractive index with respect to $C_{i}{ }^{24}$

For each set of concentrations, we report our data on the corresponding ternary diffusion coefficients, $\left(D_{i j}\right)_{V}^{23)}$, for the PEG2000 $+\mathrm{NaCl}+$ water system in Table 2. As previously mentioned, ternary diffusion coefficients for the other corresponding systems lysozyme $+\mathrm{NaCl}+$ water, $\left(D_{i j}\right)_{V}^{(13)}$, and lysozyme + PEG2000 + water, $\left(D_{i j}\right)_{V}^{(12)}$, have been previously reported. ${ }^{23,34}$ We compare $\left(D_{i j}\right)_{V}$ with $\left(D_{i j}\right)_{V}^{(13)},\left(D_{i j}\right)_{V}^{(12)}$, and $\left(D_{i j}\right)_{V}^{(23)}$ ternary data to assess prediction of quaternary diffusion coefficients from the ternary diffusion data. In each comparison, those solutes that appear in both the ternary and quaternary systems have the same concentration. 
TABLE 2: Ternary Diffusion Data for PEG2000 (2) $+\mathrm{NaCl}(3)+\mathrm{H}_{2} \mathrm{O}$

$\begin{array}{ll}\bar{C}_{2}(\mathrm{M}) & 0.04999 \\ \bar{C}_{3}(\mathrm{M}) & 0.4999 \\ \bar{d}\left(\mathrm{~g} \mathrm{~cm}^{-3}\right) & 1.03345_{8} \\ H_{2}\left(\mathrm{~kg} \mathrm{~mol}^{-1}\right) & 0.328_{6} \\ H_{3}\left(\mathrm{~kg} \mathrm{~mol}^{-1}\right) & 0.0394_{8} \\ \bar{V}_{0}\left(\mathrm{dm}^{3} \mathrm{~mol}^{-1}\right) & 0.01806 \\ \bar{V}_{2}\left(\mathrm{dm}^{3} \mathrm{~mol}^{-1}\right) & 1.676 \\ \bar{V}_{3}\left(\mathrm{dm}^{3} \mathrm{~mol}^{-1}\right) & 0.0190_{1} \\ R_{2}\left(\mathrm{M}^{-1}\right) & 12300 \\ R_{3}\left(\mathrm{M}^{-1}\right) & 442.5 \\ \lambda_{2}\left(10^{-9} \mathrm{~m}^{2} \mathrm{~s}^{-1}\right) & 0.1847 \\ \lambda_{3}\left(10^{-9} \mathrm{~m}^{2} \mathrm{~s}^{-1}\right) & 1.117 \\ \left(D_{22}\right)_{V}^{(23)}\left(10^{-9} \mathrm{~m}^{2} \mathrm{~s}^{-1}\right) & 0.2040 \pm 0.0001 \\ \left(D_{23}\right)_{V}^{(23)}\left(10^{-9} \mathrm{~m}^{2} \mathrm{~s}^{-1}\right) & 0.0085 \pm 0.00002 \\ \left(D_{32}\right)_{V}^{(23)}\left(10^{-9} \mathrm{~m}^{2} \mathrm{~s}^{-1}\right) & 2.08 \pm 0.01 \\ \left(D_{33}\right)_{V}^{(23)}\left(10^{-9} \mathrm{~m}^{2} \mathrm{~s}^{-1}\right) & 1.075 \pm 0.004 \\ \left(D_{22}\right)_{0}^{(23)}\left(10^{-9} \mathrm{~m}^{2} \mathrm{~s}^{-1}\right) & 0.2250 \\ \left(D_{23}\right)_{0}^{(23)}\left(10^{-9} \mathrm{~m}^{2} \mathrm{~s}^{-1}\right) & 0.01044 \\ \left(D_{32}\right)_{0}^{(23)}\left(10^{-9} \mathrm{~m}^{2} \mathrm{~s}^{-1}\right) & 2.29 \\ \left(D_{33}\right)_{0}^{(23)}\left(10^{-9} \mathrm{~m}^{2} \mathrm{~s}^{-1}\right) & 1.117\end{array}$

Examination of Protein Diffusion. In Figure 1, we plot the lysozyme main-term diffusion coefficient, $\left(D_{11}\right)_{V}$, as a function of $C_{2}$ and $C_{3}$. We first examine the behavior of this diffusion coefficient for the two lysozyme ternary aqueous systems (i.e., $\left(D_{11}\right)_{V}^{(13)}$ and $\left.\left(D_{11}\right)_{V}^{(12)}\right)$. We see that $\left(D_{11}\right)_{V}$ significantly decreases as either salt concentration or PEG2000 concentration increases. The large value of protein diffusion coefficient at $C_{2}=C_{3}=0$ is due to the long-range electrostatic drag of the fast chloride counterions on the lysozyme cations. ${ }^{23}$

In the lysozyme-salt case, most of the observed decrease in $\left(D_{11}\right)_{V}^{(13)}$ occurs within very low values of $C_{3}$ due to electrostatic screening. ${ }^{23,30,31}$ However in the lysozyme-polymer case, the observed decrease in $\left(D_{11}\right)_{V}^{(12)}$ is more gradual and can be largely attributed to the corresponding increase of solution viscosity, though dielectric effects on the lysozyme-chloride electrostatic interactions may also play an important role. ${ }^{33}$ Figure 1 also shows that the behavior of the quaternary data is consistent with the ternary data. However, since electrostatic screening is the dominant effect, the quaternary $\left(D_{11}\right)_{V}$ values are closer to the corresponding $\left(D_{11}\right)_{V}^{(13)}$ values than to the $\left(D_{11}\right)_{V}^{(12)}$ values within our experimental concentration domain. Thus, it is expected that

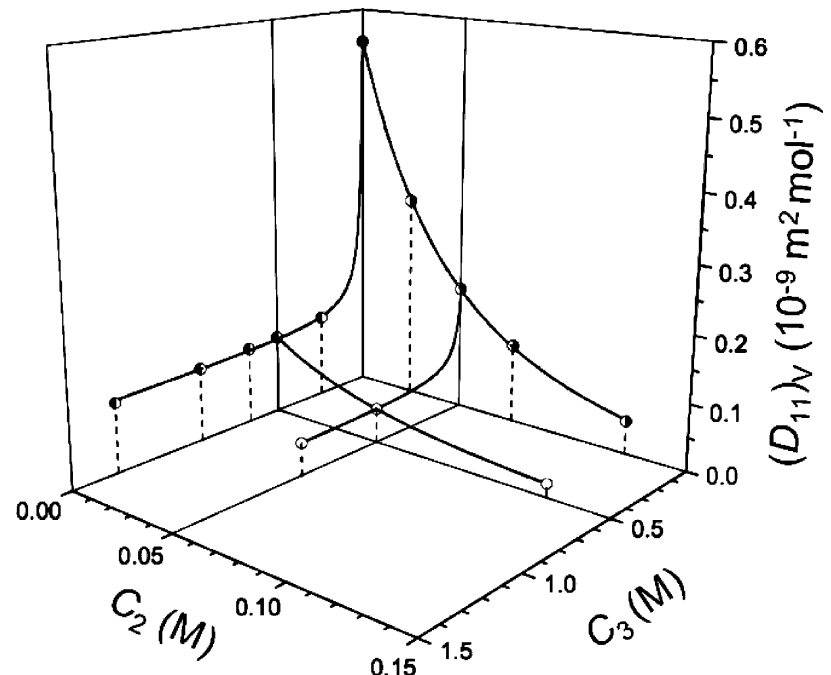

Figure 1. Lysozyme main-diffusion coefficient, $\left(D_{11}\right)_{V}$, as a function of PEG2000 concentration, $C_{2}$, and $\mathrm{NaCl}$ concentration, $C_{3}$, at $\mathrm{pH} 4.5$ and $25{ }^{\circ} \mathrm{C}$. Quaternary data points $(\mathrm{O})$ are shown together with previously reported ternary data for the lysozyme-PEG2000-water (O) and lysozyme- $\mathrm{NaCl}$-water (O) systems.

$\begin{array}{ll}0.04997 & 0.1248 \\ 0.8995 & 0.4992 \\ 1.04901_{5} & 1.05789_{1} \\ 0.310_{7} & 0.325_{9} \\ 0.0387_{9} & 0.0387_{6} \\ 0.01804 & 0.01805 \\ 1.69_{2} & 1.67_{8} \\ 0.0196_{8} & 0.0197_{3} \\ 12280 & 12460 \\ 429.8 & 431.3 \\ 0.1695 & 0.1884 \\ 1.128 & 0.703 \\ 0.1998 \pm 0.0002 & 0.2349 \pm 0.0001 \\ 0.00801 \pm 0.00008 & 0.0152 \pm 0.0002 \\ 3.51 \pm 0.04 & 1.44 \pm 0.02 \\ 1.084 \pm 0.005 & 0.648 \pm 0.008 \\ 0.2225 & 0.3024 \\ 0.00997 & 0.0213 \\ 3.92 & 1.71 \\ 1.133 & 0.682\end{array}$

TABLE 3: Ternary Diffusion and Relative Viscosity Data

\begin{tabular}{cccccc}
\hline $\begin{array}{c}C_{2} \\
(\mathrm{M})\end{array}$ & $\begin{array}{c}C_{3} \\
(\mathrm{M})\end{array}$ & $\begin{array}{c}\exp \left(D_{11}\right)_{V} \\
\left(10^{-9} \mathrm{~m}^{2} \mathrm{~s}^{-1}\right)\end{array}$ & $\begin{array}{c}\left(D_{11}\right)(13) \\
\left(10^{-9} \mathrm{~m}^{2} \mathrm{~s}^{-1}\right)\end{array}$ & $\eta_{\mathrm{r}}^{(3)} / \eta_{\mathrm{r}}^{(23)}$ & $\begin{array}{c}\text { calc }\left(D_{11}\right)_{V} \\
\left(10^{-9} \mathrm{~m}^{2} \mathrm{~s}^{-1}\right)\end{array}$ \\
\hline 0.050 & 0.5 & 0.0515 & 0.1182 & 2.27 & 0.0520 \\
0.050 & 0.9 & 0.0481 & 0.1102 & 2.23 & 0.0495 \\
0.125 & 0.5 & 0.0200 & 0.1182 & 6.65 & 0.0178
\end{tabular}

more accurate predictions of $\left(D_{11}\right)_{V}$ should be based on $\left(D_{11}\right)_{V}^{(13)}$ and not $\left(D_{11}\right)_{V}^{(12)}$.

Since lysozyme-chloride electrostatic interaction in the presence of $\mathrm{NaCl}$ is screened, dielectric effects due to PEG2000 become much less significant. Hence, the dominant effect of PEG2000 on $\left(D_{11}\right)_{V}$ is expected to be viscosity. Because lysozyme molecules are significantly larger than PEG2000 coils, we may apply a viscosity correction based on the Stokes-Einstein equation. ${ }^{48}$ In other words, we can relate the difference between $\left(D_{11}\right)_{V}$ and $\left(D_{11}\right)_{V}^{(13)}$ to the corresponding difference in viscosity of the medium surrounding the protein molecules, assumed to be a continuum. We therefore obtain the following:

$$
\left(D_{11}\right)_{V}=\left(D_{11}\right)_{V}^{(13)}\left(\eta_{\mathrm{r}}^{(3)} / \eta_{\mathrm{r}}^{(23)}\right)
$$

where $\eta_{\mathrm{r}}^{(3)}$ and $\eta_{\mathrm{r}}^{(23)}$ are relative viscosities for the PEG2000-water binary and PEG2000- $\mathrm{NaCl}$-water ternary systems, respectively.

In Table 3, we report our analysis based on eq 2. For the two cases at $C_{2}=0.05 \mathrm{M}(100 \mathrm{~g} / \mathrm{L})$, the predicted values are in good agreement with the experimental ones. A good agreement was also observed in the case of the lysozyme-TEG$\mathrm{NaCl}-$ water system with the same mass concentration of PEG. However, the calculated value of $\left(D_{11}\right)_{V}$ at $C_{2}=0.125 \mathrm{M}(250$ $\mathrm{g} / \mathrm{L})$ is $12 \%$ larger than the experimental value in the PEG2000 case (see Table 3 ). This appreciable discrepancy indicates that the approximation of treating the PEG2000- NaCl-water medium as a continuum fluid may be poorer at higher PEG2000 concentrations and should be reassessed. Clearly, the use of the viscosity correction to predict protein diffusion coefficients in highly crowded environments may not be sufficiently accurate.

A recent theoretical investigation by Tuinier et al. ${ }^{49}$ suggests that the friction experienced by globular proteins when they move through a crowded medium is reduced compared to Stokes's law due to entropic depletion effects. In other words, depletion of polymer from the protein surface leads to a lowviscosity layer around the protein as compared to the viscosity 

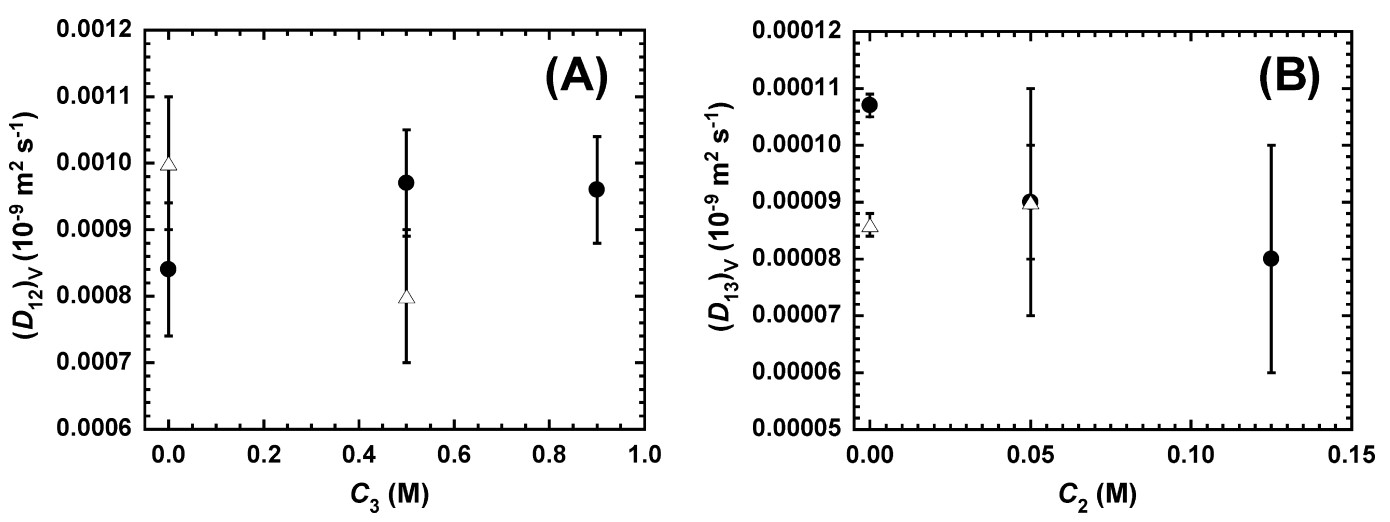

Figure 2. (A) Lysozyme cross-diffusion coefficient, $\left(D_{12}\right)_{V}$, as a function of $\mathrm{NaCl}$ concentration, $C_{3}$, at $C_{2}=0.050 \mathrm{M}(\bullet)$ and $C_{2}=0.125 \mathrm{M}(\Delta)$. (B) Lysozyme cross-diffusion coefficient, $\left(D_{13}\right)_{V}$, as a function of PEG2000 concentration, $C_{2}$, at $C_{3}=0.50 \mathrm{M}(\bullet)$ and $C_{3}=0.90 \mathrm{M}(\Delta)$. Points at $C_{3}=0(\mathrm{~A})$ and at $C_{2}=0(\mathrm{~B})$ represent $\left(D_{12}\right) V_{V}^{(12)}$ and $\left(D_{13}\right)_{V}^{(13)}$, respectively.

in the bulk solution. Our experimental results are consistent with these theoretical findings.

We now consider lysozyme cross-term diffusion coefficients, $\left(D_{12}\right)_{V}$ and $\left(D_{13}\right)_{V}$. In Figure 2(A and B), we plot $\left(D_{12}\right)_{V}$ and $\left(D_{13}\right)_{V}$ as a function of $C_{3}$ and $C_{2}$, respectively. For comparison, $\left(D_{12}\right)_{V}^{(12)}$ and $\left(D_{13}\right)_{V}^{(13)}$ of the corresponding ternary systems are also included. From the error bar comparison along the $C_{3}$ coordinate, we can see that $\left(D_{12}\right)_{V}$ and $\left(D_{13}\right)_{V}$ are approximately independent of $C_{3}$ and $C_{2}$, respectively, within the experimental error.

We note that these two cross terms, relating the fluxes of the protein to gradients of each of the other solutes, are proportional to the protein mobility ${ }^{30,34}$ and a driving force produced by the other solute gradients. Thus, as for the case of $\left(D_{11}\right)_{V}$ in eq 2 , viscosity is expected to contribute to the behavior of $\left(D_{12}\right)_{V}$ and $\left(D_{13}\right)_{V}$. In the case of $\left(D_{12}\right)_{V}$, the effect of $\mathrm{NaCl}$ concentration on solution viscosity is small. Indeed $\eta_{\mathrm{r}}^{(23)} / \eta_{\mathrm{r}}^{(2)}$ was calculated to be just 1.02 at these compositions. This implies that the viscosity effect on $\left(D_{12}\right)_{V}$ is expected to be small. On the other hand, the large values of $\eta_{\mathrm{r}}^{(23)} / \eta_{\mathrm{r}}^{(3)}$ in Table 3 would lead to predicted values of $\left(D_{13}\right)_{V}$ significantly smaller than observed for the corresponding experimental quaternary results. We therefore conclude that viscosity corrections do not account for the observed differences in $\left(D_{13}\right)_{V}$ between ternary and quaternary data. Clearly, other factors such as change in solute solvation and electrostatic interactions may be significant in $\left(D_{13}\right)_{V}$.

It is important to remark that examination of $\left(D_{13}\right)_{V}^{(13)}$ based on irreversible thermodynamics shows that the small value of this ternary cross-diffusion coefficient is the result of the difference between two relatively large terms of comparable magnitude. ${ }^{30}$ Hence, even a small perturbation to these terms can affect the value of $\left(D_{13}\right)_{V}$ in such a way that its dependence on $C_{3}$ no longer correlates with the corresponding change in viscosity.

We also note that the addition of salt to the lysozymePEG2000-water system has a significant effect on the lysozyme main-diffusion coefficient $\left(D_{11}\right)_{V}$. On the other hand, the effect of salt on the lysozyme cross term $\left(D_{12}\right)_{V}$ is found to be negligible. Hence, electrostatic screening does not affect lysozyme diffusion under a polymer concentration gradient.

Finally, the values of the two cross terms obtained at $C_{2}=$ $0.050 \mathrm{M}$ and $C_{3}=0.50 \mathrm{M}$ were also compared with those obtained for the lysozyme-TEG- $\mathrm{NaCl}$-water system in the same experimental conditions (see Table 2 of ref 24). The comparison was performed by converting each cross term, $\left(D_{i j}\right)_{V}$, to its corresponding value, $\left(D_{i j}\right)_{V}\left(M_{i} / M_{j}\right)$, in the mass-concentra-

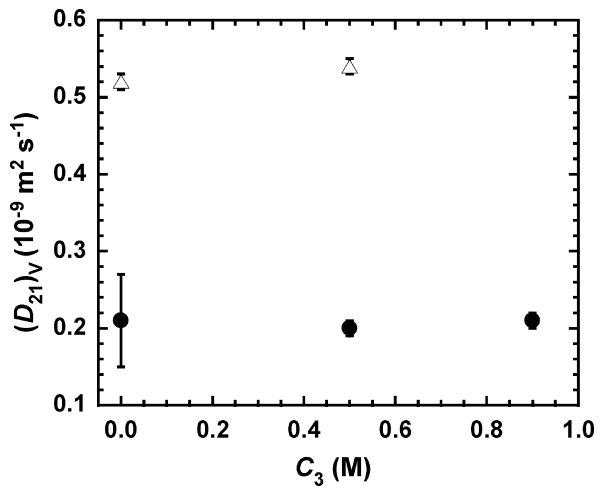

Figure 3. PEG2000 cross-diffusion coefficient, $\left(D_{21}\right)_{V}$, as a function of $\mathrm{NaCl}$ concentration, $C_{3}$, at $C_{2}=0.050 \mathrm{M}(\bullet)$ and $C_{2}=0.125 \mathrm{M}$ $(\Delta)$. Points at $C_{3}=0$ represent $\left(D_{21}\right) V_{V}^{(12)}$.

tion scale, ${ }^{50}$ where $M_{i}$ is the molar mass of component $i$. As expected, they were found to coincide fairly well within the experimental error.

Examination of Polymer Diffusion. We now consider $\left(D_{22}\right)_{V}$ and $\left(D_{23}\right)_{V}$ in Table 1 . Since the lysozyme concentration is small, we can compare these two quaternary diffusion coefficients with the corresponding ternary values in Table 2. The main-term $\left(D_{22}\right)_{V}$ is about $2 \%$ lower than the corresponding $\left(D_{22}\right)_{V}^{(23)}$ value. This result is consistent with the presence of a small obstruction effect due to the protein molecules. Since $C_{1}$ is small, the cross term, $\left(D_{23}\right)_{V}$, coincides with $\left(D_{23}\right)_{V}^{(23)}$ within the experimental error as expected.

We next compare $\left(D_{21}\right)_{V}$ for the flux of PEG2000 due to a gradient of lysozyme with the corresponding ternary data, $\left(D_{21}\right)_{V}^{(12)}$. Our results are shown in Figure 3. From the error bar comparison along the $C_{3}$ coordinate, we observe that $\left(D_{21}\right)_{V}$ and $\left(D_{21}\right)_{V}^{(12)}$ are equal to each other within the experimental error. We therefore conclude that the addition of salt has negligible effect on both lysozyme-PEG2000 cross-diffusion coefficients.

It is important to note that $\left(D_{21}\right)_{V}$ is related to the lysozymePEG2000 thermodynamic interaction. ${ }^{34}$ The effect of PEG on protein solutions is often described in terms of excluded-volume interactions between the two macromolecules. ${ }^{34,37-40}$ These interactions are responsible for the phase separation of protein solutions and stabilize the protein native form with respect to unfolded states. Since the presence of $\mathrm{NaCl}$ has no detectable effects on $\left(D_{21}\right)_{V}$, we conclude that this salt does not significantly affect lysozyme-PEG2000 thermodynamic interactions within our experimental concentration domain.

Finally, we examine the effect of PEG molecular weight on $\left(D_{21}\right)_{V}$ by comparing our results at $C_{2}=0.050 \mathrm{M}$ and $C_{3}=$ 


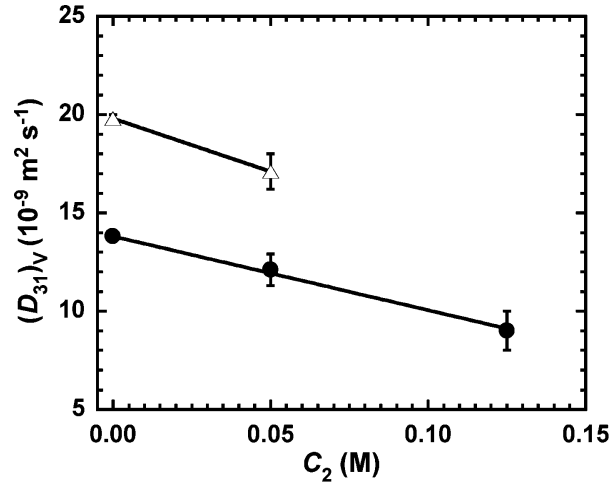

Figure 4. $\mathrm{NaCl}$ cross-diffusion coefficient, $\left(D_{31}\right)_{V}$, as a function of PEG2000 concentration, $C_{2}$, at $C_{3}=0.50 \mathrm{M}(\bullet)$ and $C_{3}=0.90 \mathrm{M}(\Delta)$. Points at $C_{2}=0$ represent $\left(D_{31}\right)_{V}^{(13)}$.

$0.50 \mathrm{M}$ with those obtained for the lysozyme-TEG-NaCl-water system. In limit of dilute solutions, $\left(D_{21}\right)_{V}$ becomes directly proportional to both $C_{2}$ and $\left(D_{22}\right)_{V} \cdot{ }^{34}$ Thus, a meaningful comparison must be performed by considering the quotient: $\left(D_{21}\right)_{V} /\left[C_{2}\left(D_{22}\right)_{V}\right]$. This quotient was found to be $14 \pm 1 \mathrm{M}^{-1}$ in the TEG case and $20 \pm 1 \mathrm{M}^{-1}$ in the PEG2000 case. The observed increase of $\left(D_{21}\right)_{V} /\left[C_{2}\left(D_{22}\right)_{V}\right]$ with PEG molecular weight is consistent with a corresponding increase in lysozyme-PEG repulsive interactions.

Examination of Salt Diffusion. We now consider $\left(D_{33}\right)_{V}$ and $\left(D_{32}\right)_{V}$ in Table 1 . As in the case of $\left(D_{22}\right)_{V}$ and $\left(D_{23}\right)_{V}$, we expect small changes with respect to the corresponding ternary values due to the low protein concentration. Indeed, we find that (1) $\left(D_{33}\right)_{V}$ is about $2 \%$ lower than the corresponding $\left(D_{33}\right)_{V}^{(23)}$ value due to obstruction effects ${ }^{23}$ and (2) $\left(D_{32}\right)_{V}$ coincides with $\left(D_{32}\right)_{V}^{(23)}$ within the experimental error.

Finally, we consider the cross term, $\left(D_{31}\right)_{V}$, for the flux of $\mathrm{NaCl}$ due to a gradient of lysozyme. Our results are shown in Figure 4 together with the previously reported $\left(D_{31}\right)_{V}^{(13)}$. As previously discussed for the lysozyme- $\mathrm{NaCl}$-water ternary system, ${ }^{23}$ the large values of $\left(D_{31}\right)_{V}^{(13)}$ indicate a significant flux of salt induced in the presence of protein concentration gradients. At low salt concentration, the value of $\left(D_{31}\right)_{V}^{(13)}$ can be mainly attributed to a common-ion effect. Furthermore, $\left(D_{31}\right)_{V}^{(13)}$ linearly increases with salt concentration. This phenomenon has been related to an excluded-volume effect. ${ }^{31}$

As shown in Figure $4,\left(D_{31}\right)_{V}$ decreases as $C_{2}$ increases. This behavior can be related to the large obstruction effect of PEG2000 molecules on the mobility of the salt ions. For the ternary lysozyme $-\mathrm{NaCl}$-water system, we have shown that the behavior of this cross term is better examined by considering the solvent-frame ratio $\left(D_{31}\right)_{0}^{(13)} /\left(D_{33}\right)_{0}^{(13)}$. $^{31}$ This quotient is essentially a thermodynamic quantity. Indeed, we can write the following approximate equation: ${ }^{26}$

$$
\frac{\left(D_{31}\right)_{0}^{(13)}}{\left(D_{33}\right)_{0}^{(13)}} \approx-\left(\frac{\partial C_{3}}{\partial C_{1}}\right)_{\mu_{3}, T, p}=\frac{\mu_{31}^{(13)}}{\mu_{33}^{(13)}}
$$

where $\mu_{i j} \equiv\left(\partial \mu_{i} / \partial C_{j}\right)_{T, p, C_{k}, k \neq j}, T$ is the temperature, $p$ is the pressure, and the superscript $(i j)$ appended to the chemicalpotential derivative identifies the solute pair of the ternary system in the same way as shown for the diffusion coefficients.

This result can be extended to $\left(D_{31}\right)_{0} /\left(D_{33}\right)_{0}$ for our quaternary system. To understand the thermodynamic nature of this ratio, we consider multicomponent diffusion occurring between two solutions having same $\mathrm{NaCl}$ and PEG2000 concentrations and different lysozyme concentration. Since diffusion of lysozyme in the presence of its own concentration gradient is relatively slow, the fast mutual diffusion between the $\mathrm{NaCl}$ and water components will lead to a fast but changing quasi-equilibrium condition, $\left(J_{3}\right)_{0} \approx 0$, in the presence of a much slower dissipating concentration gradient of lysozyme. Therefore the diffusion of lysozyme can be neglected. We note that diffusion of PEG2000 can also be neglected in these conditions because (1) there is no initial PEG2000 concentration gradient driving its flux; (2) its diffusion is slow compared to $\mathrm{NaCl}$, though not as slow as lysozyme. According to eq 1 in the solvent frame, $\left(J_{3}\right)_{0} \approx 0$ together with $\nabla C_{2} \approx 0$ yields $\left(D_{31}\right)_{0} \nabla C_{1} \approx-\left(D_{33}\right)_{0} \nabla C_{3}$. This result can be rewritten as

$$
\frac{\left(D_{31}\right)_{0}}{\left(D_{33}\right)_{0}} \approx-\left(\frac{\partial C_{3}}{\partial C_{1}}\right)_{T, p, \mu_{3}, C_{2}}=\frac{\mu_{31}}{\mu_{33}}
$$

In Table 4, we compared $\left(D_{31}\right)_{0}^{(13)} /\left(D_{33}\right)_{0}^{(13)}$ with $\left(D_{31}\right)_{0} /\left(D_{33}\right)_{0}$. We see that the quaternary-diffusion ratios are larger than the corresponding ternary ratios. The same result is also obtained for the corresponding volume-frame ratios (not shown). Thus, PEG2000 enhances salt diffusion in the presence of protein concentration gradients. Since this effect was not observed in the lysozyme-TEG- $\mathrm{NaCl}$-water system, ${ }^{24}$ our results suggest that $\left(D_{31}\right)_{0} /\left(D_{33}\right)_{0}$ also increases with PEG molecular weight at a given polymer mass concentration.

As shown in Figure 5, the change of $\left(D_{31}\right)_{0} /\left(D_{33}\right)_{0}$ with PEG2000 concentration can be described by a linear increase. To explain the experimental behavior, we now derive an expression for $\left(D_{31}\right)_{0} /\left(D_{33}\right)_{0}$ based on thermodynamic examination of $\mu_{31} / \mu_{33}$. Since molality-based cross derivatives of chemical potentials are equal, the following thermodynamic relations can be written for the ternary (eq 5) and quaternary (eq 6) systems: ${ }^{31,50}$

$$
\frac{\mu_{31}^{(13)}}{\mu_{33}^{(13)}}=\frac{\mu_{13}^{(13)}}{\mu_{33}^{(13)}} \frac{1-C_{3} \bar{V}_{3}}{1-C_{1} \bar{V}_{1}}-\frac{\mu_{11}^{(13)}}{\mu_{33}^{(13)}} \frac{C_{1} \bar{V}_{3}}{1-C_{1} \bar{V}_{1}}+\frac{C_{3} \bar{V}_{1}}{1-C_{1} \bar{V}_{1}}
$$

$$
\begin{array}{r}
\frac{\mu_{31}}{\mu_{33}}=\frac{\mu_{13}}{\mu_{33}} \frac{1-C_{3} \bar{V}_{3}}{1-C_{1} \bar{V}_{1}}-\frac{\mu_{11}}{\mu_{33}} \frac{C_{1} \bar{V}_{3}}{1-C_{1} \bar{V}_{1}}-\frac{\mu_{12}}{\mu_{33}} \frac{C_{2} \bar{V}_{3}}{1-C_{1} \bar{V}_{1}}+ \\
\frac{\mu_{32}}{\mu_{33}} \frac{C_{2} \bar{V}_{1}}{1-C_{1} \bar{V}_{1}}+\frac{C_{3} \bar{V}_{1}}{1-C_{1} \bar{V}_{1}}
\end{array}
$$

We now observe that all $C_{i} \bar{V}_{j}$ terms in eqs 5 and 6 with the exception of $C_{2} \bar{V}_{1}$ and $C_{3} \bar{V}_{1}$ are small compared to unity. We can therefore obtain the following approximate relations:

$$
\begin{gathered}
\frac{\mu_{31}^{(13)}}{\mu_{33}^{(13)}} \approx \frac{\mu_{13}^{(13)}}{\mu_{33}^{(13)}}+C_{3} \bar{V}_{1} \text { ternary } \\
\frac{\mu_{31}}{\mu_{33}} \approx \frac{\mu_{13}}{\mu_{33}}+\frac{\mu_{32}}{\mu_{33}} C_{2} \bar{V}_{1}+C_{3} \bar{V}_{1} \quad \text { quaternary }
\end{gathered}
$$

We now note that $\left(D_{32}\right)_{0}^{(23)} /\left(D_{33}\right)_{0}^{(23)}$ for the PEG2000- $\mathrm{NaCl}-$ water system can be also approximated by the corresponding thermodynamic ratio, $\mu_{32}^{(23)} / \mu_{33}^{(33)}$, as shown for $\left(D_{31}\right)_{0}^{(13)} /\left(D_{33}\right)_{0}^{(13)}$ 
TABLE 4: Experimental and Calculated Solvent-Frame Diffusion Ratios

\begin{tabular}{cccccccc}
\hline$C_{2}(\mathrm{M})$ & $C_{3}(\mathrm{M})$ & $\left(D_{31}\right)_{0} /\left(D_{33}\right)_{0}$ & $\left(D_{31}\right)_{0}^{(13)} /\left(D_{33}\right)_{0}^{(13)}$ & $\left(D_{32}\right)_{0}^{(23)} /\left(D_{33}\right)_{0}^{(23)}$ & $(\mathrm{calc})\left(D_{31}\right)_{0} /\left(D_{33}\right)_{0}$ & $\left(D_{31}\right)_{0} /\left(D_{11}\right)_{0}$ & $\left(D_{31}\right)_{0}^{(13)} /\left(D_{11}\right)_{0}^{(13)}$ \\
\hline 0.050 & 0.5 & $11.6 \pm 0.8$ & $9.9 \pm 0.2$ & $2.05 \pm 0.01$ & $10.9 \pm 0.2$ & $230 \pm 20$ \\
0.050 & 0.9 & $16.3 \pm 0.8$ & $14.2 \pm 0.2$ & $3.52 \pm 0.04$ & $16.0 \pm 0.2$ & $370 \pm 20$ \\
0.125 & 0.5 & $15 \pm 1$ & $9.9 \pm 0.2$ & $2.51 \pm 0.03$ & $13.1 \pm 0.2$ & $480 \pm 50$
\end{tabular}

in eq 3. Moreover, since the lysozyme concentration is small, $\mu_{32} / \mu_{33}$ in eq 8 is essentially equal to $\mu_{32}^{(23)} / \mu_{33}^{(33)}$. We also note that $\mu_{13}^{(13)} / \mu_{33}^{(13)}$ is approximately equal to the molality-based chemical-potential ratio, ${ }^{28}$ which is directly related to the thermodynamic interaction between two different solutes according to preferential-interaction theory. ${ }^{51,52}$ If the protein-salt thermodynamic interaction is not significantly affected by the presence of PEG2000, we can approximate $\mu_{13} / \mu_{33}$ with the corresponding $\mu_{13}^{(13)} / \mu_{33}^{(13)}$. Thus, by combining eqs 4,7 , and 8 , we finally obtain the following:

$$
\frac{\left(D_{31}\right)_{0}}{\left(D_{33}\right)_{0}} \approx \frac{\left(D_{31}\right)_{0}^{(13)}}{\left(D_{33}\right)_{0}^{(13)}}+\frac{\left(D_{32}\right)_{0}^{(23)}}{\left(D_{33}\right)_{0}^{(23)}} C_{2} \bar{V}_{1}
$$

A similar expression can be also derived for $\left(D_{32}\right)_{0} /\left(D_{33}\right)_{0}$ provided that $C_{2}$ is small. Since $\left(D_{32}\right)_{0}^{(23)} /\left(D_{33}\right)_{0}^{(23)}$ is a positive quantity, eq 9 correctly predicts an increase of $\left(D_{31}\right)_{0} /\left(D_{33}\right)_{0}$ with PEG2000 concentration. In Table 4 , we report $\left(D_{31}\right)_{0} /\left(D_{33}\right)_{0}$ values calculated using eq 9 with $\bar{V}_{1}=10.2 \mathrm{dm}^{3} \mathrm{~mol}^{-1}$ (see ref 15 and Table 1). We see that our predictions are in good agreement with the corresponding experimental values.

An equivalent approach to estimate the quaternary ratio $\left(D_{31}\right)_{0} /$ $\left(D_{33}\right)_{0}$ from ternary data can be obtained by defining multicomponent diffusion coefficients with respect to molality concentration gradients as shown in ref 26. The value of $\left(D_{31}\right)_{0} /\left(D_{33}\right)_{0}$ can be then calculated by assuming that molality-based diffusion coefficients are independent of PEG concentration.

Effect of Cross-Diffusion on Protein Crystallization. The effect of cross-diffusion coefficients on protein crystallization processes has been examined in model calculations. ${ }^{27,24}$ In relation to crystal growth, diffusion is driven mainly by the depletion of protein at the crystal surface with respect to the bulk solution. Here the cross terms $\left(D_{31}\right)_{V}$ and $\left(D_{21}\right)_{V}$, which have been found to be positive, characterize salt and polymer transport toward the crystal induced by the protein concentration gradient. These calculations have shown that the concentration of precipitant (salt or polymer) at the crystal surface is enhanced if the precipitant cross-term diffusion coefficient is large compared to precipitant and protein main-term diffusion coefficients.

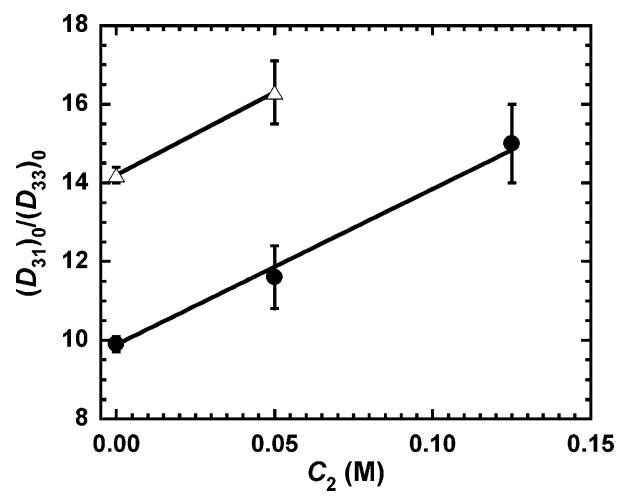

Figure 5. $\mathrm{NaCl}$ diffusion-coefficient ratio, $\left(D_{31}\right)_{0} /\left(D_{33}\right)_{0}$, as a function of PEG2000 concentration, $C_{2}$, at $C_{3}=0.50 \mathrm{M}(\bullet)$ and $C_{3}=0.90 \mathrm{M}$ $(\Delta)$. Points at $C_{2}=0$ represent $\left(D_{31}\right)_{0}^{(31)} /\left(D_{33}\right)_{0}^{(31)}$.
We now note that $\left(D_{31}\right)_{V} /\left(D_{33}\right)_{V}$ is larger than the corresponding ternary value, $\left(D_{31}\right)_{0}^{(13)} /\left(D_{33}\right)_{0}^{(13)}$ and $\left(D_{31}\right)_{V} /\left(D_{11}\right)_{V}$ is significantly larger than $\left(D_{31}\right)_{0}^{(13)} /\left(D_{11}\right)_{0}^{(13)}$. Thus, our experimental results and theoretical analysis suggest that salt concentration at the crystal surface is significantly enhanced in the presence of PEG.

Comments on Dynamic Light Scattering. Protein diffusion coefficients have been extensively measured by dynamic light scattering (DLS) ${ }^{53}$ However, in multicomponent mixtures, this technique only provides some of the eigenvalues of the diffusion coefficient matrix. ${ }^{54-56}$ Since cross-diffusion coefficients cannot be directly determined, each measurable eigenvalue is often assumed to be equal to a corresponding main-term diffusion coefficient.

We can use our experimental values of $\left(D_{11}\right)_{V}$ and the lowest eignevalue, $\lambda_{1}$, of the diffusion coefficient matrix (see Table 1) to test the assumption that DLS measurements on protein diffusion lead to $\left(D_{11}\right)_{V}$ in crowded environments. We find that, while the experimental ternary diffusion coefficient, $\left(D_{11}\right)_{V}^{(13)}$, is only $1 \%$ higher than the $\lambda_{1}$ (see Table 7 of ref 23 ), the corresponding quaternary value $\left(D_{11}\right)_{V}$ at the highest experimental PEG concentration is $13 \%$ higher. Thus, significant discrepancies between $\left(D_{11}\right)_{V}$ and DLS data may occur in multicomponent crowded systems.

\section{Summary and Conclusions}

We have successfully characterized multicomponent diffusion in the lysozyme-PEG2000- $\mathrm{NaCl}$-water quaternary system by precision Rayleigh interferometry. Our experimental work represents the first rigorous experimental investigation of diffusion in protein-polymer-salt-water systems with a significantly higher molecular-weight polymer.

We have found that $\left(D_{11}\right)_{V}$ decreases as PEG2000 concentration increases at a given $\mathrm{NaCl}$ concentration. This behavior can be quantitatively related to the corresponding increase in fluid viscosity only at the lower experimental polymer concentration. At the higher polymer concentration, protein diffusion is enhanced compared to the viscosity prediction.

We have found that the addition of $\mathrm{NaCl}$ to the lysozymePEG2000-water system has no effect on $\left(D_{21}\right)_{V}$. This indicates that lysozyme-PEG2000 thermodynamic interactions are not significantly affected by the salt component. We have also found that $\left(D_{31}\right)_{0} /\left(D_{33}\right)_{0}$ increases with PEG2000 concentration. Since salt diffusion due to protein concentration gradients is enhanced in the presence of a uniform polymer concentration, this enhancement suggests that salt incorporation inside protein crystals will be facilitated in the presence of polymers.

We believe that this work represents a significant contribution to the characterization of protein diffusion in crowded environments. It may provide guidance for further theoretical developments in the field of protein transport properties relevant not only to biotechnological processes but also to understanding protein mobility inside the cytoplasm of living cells.

Acknowledgment. This research was supported by the Italian Space Agency (ARS-99-15), the NASA BioTechnology Program (NAG8-1356), and the ACS Petroleum Research Fund (47244-G4). 


\section{References and Notes}

(1) Vekilov, P. G. Cryst. Growth Des. 2007, 7, 2796-2810.

(2) Garcia-Ruiz, J. M. Meth. Enzym. 2003, 368, 130-154.

(3) Chayen, N. E. Curr. Opin. Struct. Biol. 2004, 14, 577-583. 2001.

(4) Roe, S. Protein Purification Techniques, Oxford: Oxford, U.K.,

(5) Ayala, M.; Vasquez-Duhalt, R. Enzymatic catalysis on petroleum products. In Studies in Surface Science and Catalysis; Vasquez-Duhalt, R. Quintero-Ramirez, R., Eds.; Elsevier: Amsterdam, 2004; Vol. 151, pp 67111.

(6) Roy, J. J.; Abraham, T. E. Chem. Rev. 2004, 104, 3705-3721.

(7) Miguez, D. G.; Vanag, V. K.; Epstein, I. R. Proc. Natl. Acad. Sci. U.S.A. 2007, 104, 6992-6997.

(8) Price, P. E.; Romdhane, I. H. AIChE J. 2003, 49, 309-322.

(9) Jervais, T.; Jensen, K. F. Chem. Eng. Sci. 2006, 61, 1102-1121. 663.

(11) Vekilov, P. G. Cryst. Growth Des. 2007, 7, 2239-2246.

(12) Timasheff, S. N. Proc. Natl. Acad. Sci. U.S.A. 2002, 99, 97219726.

(13) McPherson, A. Crystallization of Biological Macromolecules; Cold Spring Harbor: New York, 1998.

(14) Ducruix, A.; Giegé, R. Crystallization of nucleic acids and proteins. A practical approach; Oxford University Press: New York, 1992.

(15) Retailleau, P.; Ducruix, A.; Ries-Kautt, M. Acta Crystallogr. 2002, D58, 1576-1581.

(16) Anderson, V. J.; Lekkerkerker, H. N. W. Nature 2002, 416, 811815.

(17) Muschol, M.; Rosenberger, F. J. Chem. Phys. 1997, 107, 19531962.

(18) Vivares, D.; Bonnete, F. J. Phys. Chem. B 2004, 108, 6498-6507. (19) Annunziata, O.; Asherie, N.; Lomakin, A.; Pande, J.; Ogun, O.; Benedek, G. B. Proc. Natl. Acad. Sci. U.S.A. 2002, 99, 14165-14170.

(20) Bloustine, J.; Virmani, T.; Thurston, G. M.; Fraden, S. Phys. Rev. Lett. 2006, 96, 087803.

(21) Ru, M. T.; Hirokane, S. Y.; Lo, A. S.; Dordick, J. S.; Reimer, J. A.; Clark, D. S. J. Am. Chem. Soc. 2000, 122, 1565-1571.

(22) Tyrrell, H. J. V.; Harris, K. R. Diffusion in Liquids; Butterworths: London, 1984

(23) Albright, J. G.; Annunziata, O.; Miller, D. G.; Paduano, L.; Pearlstein, A. J. J. Am. Chem. Soc. 1999, 121, 3256-3266.

(24) Annunziata, O.; Vergara, A.; Paduano, L.; Sartorio, R.; Miller, D. G.; Albright, J. G. J. Phys. Chem. B 2003, 107, 6590-6597.

(25) Kirkwood, J. G.; Baldwin, R. L.; Dunlop, P. J.; Gosting, L. J.; Kegeles, G. J. Chem. Phys. 1960, 33, 1505-1513.

(26) Miller, D. G.; Vitagliano, V.; Sartorio, R. J. Phys. Chem. 1986 90, 1509-1519.

(27) Castagnolo, D.; Vergara, A.; Paduano, L.; Sartorio, R.; Annunziata,

O. Acta Crystallogr. 2002, D58, 1633-1637.

(28) Annunziata, O.; Albright, J. G. Ann. N.Y. Acad. Sci. 2002, 974, 610-616.
(29) Leaist, D. G. J. Phys. Chem. 1989, 93, 474-479.

(30) Annunziata, O.; Paduano, L.; Pearlstein, A. J.; Miller, D. G.; Albright, J. G. J. Am. Chem. Soc. 2000, 122, 5916-5928.

(31) Annunziata, O.; Paduano, L.; Pearlstein, A. J.; Miller, D. G.; Albright, J. G. J. Phys. Chem. B 2006, 110, 1405-1415.

(32) Annunziata, O.; Paduano, L.; Albright, J. G. J. Phys. Chem. B 2007, 111, 10591-10598.

(33) Vergara, A.; Paduano, L.; Sartorio, R. Macromolecules 2002, 35, $1389-1398$.

(34) Vergara, A.; Capuano, F.; Paduano, L.; Sartorio, R. Macromolecules 2006, 39, 4500-4506.

(35) Leaist, D. G.; Hao, L. J. Chem. Soc., Faraday Trans. 1993, 89, 2775-2782.

(36) Paduano, L.; Sartorio, R.; Vitagliano, V.; Albright, J. G.; Miller, D. G. J. Phys. Chem. 1992, 96, 7478-7483.

(37) Bhat, R.; Timasheff, S. N. Protein Sci. 1992, 1, 1133-1143.

(38) Asakura, S.; Oosawa, F. J. Chem. Phys. 1954, 22, 1255.

(39) Vivares, D.; Belloni, L; Tardieu, A.; Bonnete, F. Eur. Phys. J. E 2002, 9, 15-25.

(40) Hall, D.; Minton, A. P. Biochim. Biophys. Acta 2003, 1649, 127139.

(41) Vergara, A.; Annunziata, O.; Paduano, L.; Miller, D. G.; Albright, J. G.; Sartorio, R. J. Phys. Chem. B 2004, 108, 2764-2772.

(42) Capuano, F.; Vergara, A.; Paduano, L.; Annunziata, O.; Sartorio, R. J. Phys. Chem. B 2003, 107, 12363-12369.

(43) Vergara, A.; Paduano, L.; Vitagliano, V.; Sartorio, R. Macromolecules 2001, 34, 991-1000.

(44) Rard, J. A. J. Chem. Thermodyn. 1996, 28, 83-110.

(45) Gosting, L. J.; Kim, H.; Loewenstein, M. A.; Reinfelds, G.; Revzin, A. Rev. Sci. Instrum. 1973, 44, 1602-1609.

(46) Miller, D. G.; Albright, J. G. Optical Methods. In Measurement of the Transport Properties of Fluids: Experimental Thermodynamics; Wakeham, W. A., Nagashima, A., Sengers, J. V., Eds.; Blackwell Scientific Publications: Oxford, U.K., 1991; Vol. 3, pp 272-294.

(47) Rard, J. A.; Miller, D. G.; Albright, J. G.; Zeidler, M. E. J. Chem. Soc., Faraday Trans. 1996, 93, 4187-4197.

(48) Tanford, C. Physical Chemistry of Macromolecules; Wiley: New York, 1961.

(49) Tuinier, R.; Dhont, J. K. G.; Fan, T.-H. Europhys. Lett. 2006, 75 , 929-935.

(50) Miller, D. G. J. Phys. Chem. 1959, 63, 570-578.

(51) Arakawa, T.; Timasheff, S. N. Meth. Enzym. 1985, 114, $49-77$.

(52) Record, M. T.; Anderson, C. F. Biophys. J. 1995, 68, 786-794.

(53) Schmitz K. S. Introduction to Dynamic Light Scattering by Macromolecules; Academic Press: San Diego, 1990.

(54) Pusey, P. N.; Fijnaut, H. M.; Vrij, A. J. Chem. Phys. 1982, 77, $4270-4281$.

(55) Leaist, D. G.; Hao, L. J. Phys. Chem. 1993, 97, 7763-7768.

(56) Annunziata, O.; Buzatu, D.; Albright, J. G. Langmuir 2005, 21, 12085-12089.

JP906977M 